\title{
Acoustic Scattering from a Strained Region
}

\author{
D. Allwright ${ }^{1} \dagger$, G. Jones ${ }^{2}$ and W. PArnell ${ }^{2}$ \\ ${ }^{1}$ Smith Institute, Oxford, UK \\ ${ }^{2}$ University of Oxford
}

(Communicated to MIIR on 27 July 2021)

Study Group: ESGI 52. 21-24 March 2005, Manchester

Communicated by: Hilary Ockendon

Industrial Partner: Thales Underwater Systems

Presenter: Peter Brazier-Smith

Team Members: David Abrahams, University of Manchester; David Allwright, Smith Institute; Chris Bell, University of Oxford; Tamas Bodai, University of Aberdeen; Philip Bond, University of Oxford; Andrew Hazel, University of Manchester; Gareth Jones, University of Oxford; Roman Novokshanov, University of Oxford; David Parker, University of Edinburgh; William Parnell, University of Manchester; Emmanuel Perrey-Debain, University of Manchester; Ben Veitch, University of Manchester.

Industrial Sector: Defence

Tools: Acoustics

Key Words: Acoustics, Composite materials, Elasticity

MSC2020 Codes: 76, 78, 74

\section{Summary}

Acoustic coatings for underwater applications may be made of a composite material consisting of gas-filled microspheres embedded in a rubber-like material. Under typical applied hydrostatic pressure the microspheres are compressed to a large static deformation. The region of the rubber around each microsphere is therefore also subject to large deformation. The Study Group addressed the problem of the propagation of small amplitude acoustic waves through this pre-strained region around the microsphere. The report reviews the material models, and formulates the full scattering problem. It is also shown how the monopole scattering strength of the microsphere embedded in the pre-strained material can be calculated by using the dynamic shear modulus of the material and the solution to the static compression problem.

$\dagger$ Corresponding author. Email: allwright@maths.ox.ac.uk 


\title{
Report prepared by
}

\author{
David Allwright (Smith Institute) \\ Gareth Jones (University of Oxford) \\ William Parnell (University of Manchester)
}

\section{Introduction}

A composite material consists of a rubber filled with gas-filled microspheres. In underwater applications it is compressed hydrostatically by a pressure that may be not insignificant compared with the shear modulus of the rubber. Thus large strains are produced around each spherical inclusion. When these spherical inclusions scatter an incident acoustic wave, the strained region around an inclusion has had its elastic properties altered by the large static strain. How does this strained region affect the elastic scattering? The acoustic wavelength is large compared with the diameter of the spherical inclusions. For the purposes of this Study Group problem, the homogenization problem (obtaining the macroscopic behaviour of the bulk material from the behaviour of a single microsphere) is to be assumed solvable. So the Study Group is asked to focus on scattering by a single microsphere and the strained region around it. The particular concern that the Study Group is asked to focus on is that the material dynamic moduli are different from the static moduli, and we wish to allow properly for this in the analysis. The bulk modulus can be treated as constant, but the dynamic shear modulus of the rubber can increase by at least an order of magnitude over its static value at frequencies of interest. A typical variation with frequency at 2 different temperatures is shown below. Previous work on this problem by W.J.Parnell is in [1].

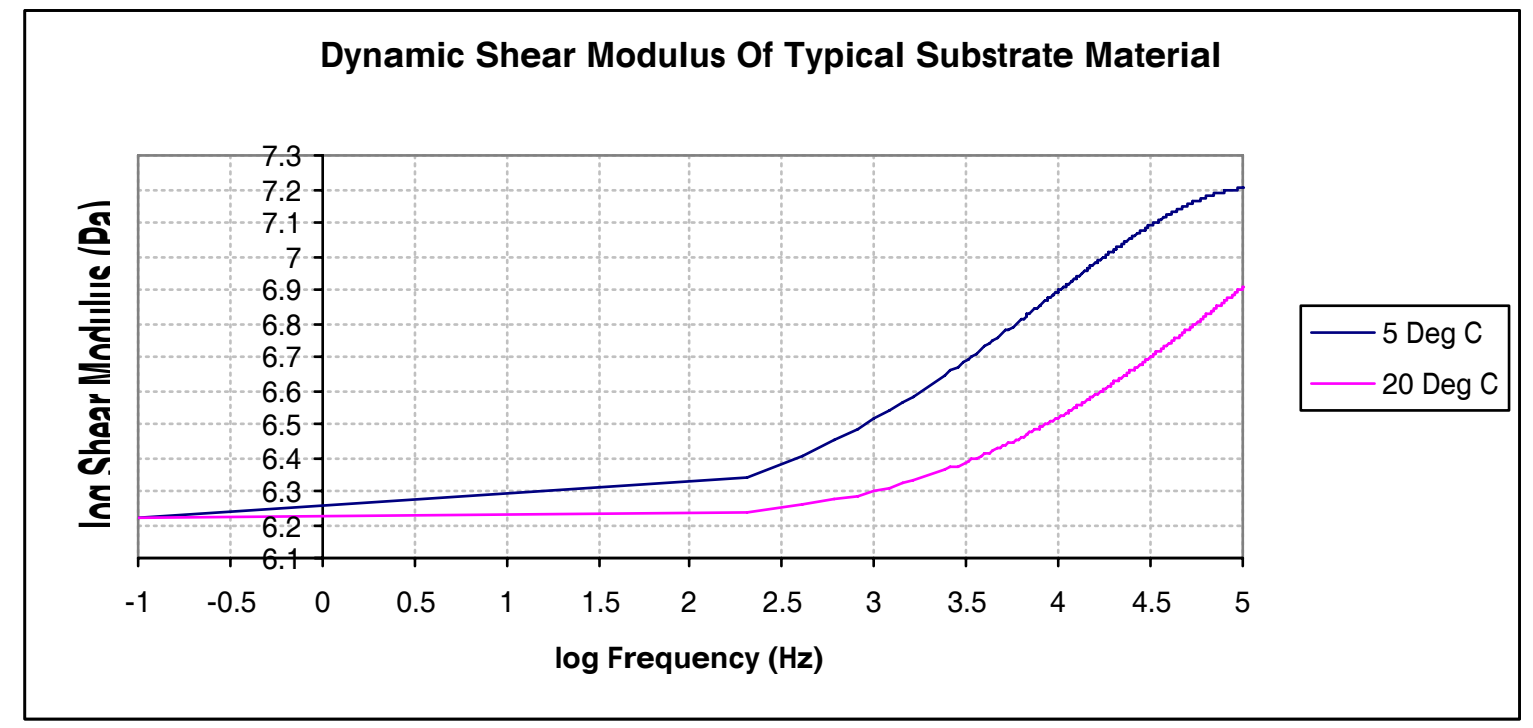

In this problem report, the work is divided up into various sections. First, there are obviously some material modelling issues: a mathematical model is needed for small 
strain dynamic viscoelasticity superimposed on a large static strain. Some of the existing models for this are reviewed in Section 2. Then, in order to formulate the scattering problem for compressive waves correctly, we need to compute the static state allowing for a finite compressibility. This is in contrast to [1], where the static state was only computed explicitly for an incompressible material, and then this was used as the basis for an approximate computation of the monopole scattering cross-section. Our calculation of the correction to this static state, to allow for finite compressibility, is in Section 3. Then in Section 4 we address the scattering problem itself.

\section{$2 \quad$ Material Modelling}

There are in the literature a number of models for dynamic large strain viscoelasticity of rubbery materials, and in particular we referred to papers by Simo [2] and by Kim, Youn and Lee [3].

Simo's model initially assumes a single time-constant $\nu$ in the viscoelastic behaviour, but this is generalized later. We initially treat the unstressed state of the rubber as the reference configuration, and let the deformation gradient matrix at any point be $F$, so $J=\operatorname{det}(F)$ is the volume ratio. The deviatoric part of the deformation is written as

$$
\bar{E}=\frac{1}{2}\left(\frac{F^{T} F}{J^{2 / 3}}-I\right)
$$

so this represents the change of shape of a material element. Then the free energy is written in the form

$$
U^{0}(J)+\bar{\Psi}_{0}(\bar{E})-Q: \bar{E}+\Psi_{I}(Q) .
$$

Here, $U^{0}(J)$ represents the term due to change of volume, and if $K_{0}$ is the bulk modulus then the correct form for small deformations is

$$
U^{0}(J)=\frac{1}{2} K_{0}(J-1)^{2} .
$$

The matrix $Q$ is an internal variable representing the relaxation process, and will be governed by a first order equation in time

$$
\frac{d Q}{d t}+\frac{Q}{\nu}=\frac{1-\gamma}{\nu} \operatorname{DEV}\left(\frac{\partial \bar{\Psi}_{0}(\bar{E})}{\partial \bar{E}}\right)
$$

where $\operatorname{DEV}($.) denotes taking the deviatoric part. In this, $0<\gamma<1$ and of course $\nu>0$.

This model has the following features:

(1) By choice of $\bar{\Psi}_{0}$ it can be matched to a given measured static large-strain behaviour.

(2) For small dynamic strains about the unstressed state, it gives a dynamic shear modulus

$$
\mu(\omega)=\mu^{0}\left(\frac{\gamma+i \omega \nu}{1+i \omega \nu}\right)
$$


where $\gamma \mu^{0}$ and $\mu^{0}$ are the rubbery and glassy shear modulus (i.e. the low frequency and high frequency limits). The transition region between these limits is where $\omega \nu=O(1)$.

(3) The model agrees well with experiments on pure rubbers. (Different models, developed in [3], are needed for "filled" rubbers, i.e. rubbers filled with, for instance, carbon particles.)

(4) The model predicts that the dynamic moduli for small deviations about a large static strain are got by taking the corresponding static moduli and replacing the static shear modulus $\mu$ by $\mu(\omega)$. For instance, suppose the (1111) static differential stiffness is $\mu \lambda_{1}$, where $\lambda_{1}$ is a principal stretch, so small static increments to strain and stress obey

$$
\delta \sigma_{11}=\mu \lambda_{1} \delta e_{11}+\ldots
$$

Then in a dynamic problem at frequency $\omega$, with $\delta e_{i j}=\Re\left(\delta \tilde{e}_{i j} e^{i \omega t}\right)$ and $\delta \sigma_{i j}=$ $\Re\left(\delta \tilde{\sigma}_{i j} e^{i \omega t}\right)$, we shall have

$$
\delta \tilde{\sigma}_{11}=\mu(\omega) \lambda_{1} \delta \tilde{e}_{11}+\ldots
$$

In other words, $\mu(\omega) \lambda_{1}$ is the nonlinear dynamic stiffness.

(5) Viscoelastic models with multiple relaxation times can be handled in the same way, and the upshot is that one replaces the factor $\mu$ in the static incremental moduli by $\mu(\omega)$ to obtain the dynamic incremental modulus. The values of this shear modulus can either be measured directly, or inferred from a measured temperaturedependence by the WLF rule in ways that are well-known.

\section{Static state: compressibility correction}

To analyse compressive waves as departures from a strained state, we must start from a strained state that allows for a finite compressibility. We therefore consider a spherical cavity of initial radius $a_{0}$, in a material with static strain energy function

$$
W=\frac{1}{2} \mu\left(\lambda_{1}^{2}+\lambda_{2}^{2}+\lambda_{3}^{2}-3-2 \log V\right)+\frac{1}{2} \lambda(V-1)^{2} .
$$

In this, $\lambda$ and $\mu$ are the usual Lamé moduli, the $\lambda_{i}$ are the principal stretches of a material element, and $V=\lambda_{1} \lambda_{2} \lambda_{3}$ is the volume ratio. This is referred to as a compressible neoHookean material. We assume that the compressed state has a uniform hydrostatic pressure $p_{\infty}$ as $r \rightarrow \infty$, a pressure $p_{\text {in }}$ in the cavity, and a compressed radius $a$. The whole deformation is spherically symmetric, so the material at a given radius $r_{0}>a_{0}$ in the unstressed state will deform to some radius $r>a$ in the stressed state, and the principal stretches $\lambda_{r}$ radially and $\lambda_{t}$ tangentially will be

$$
\lambda_{r}=\frac{d r}{d r_{0}}, \quad \lambda_{t}=\frac{r}{r_{0}} .
$$


The corresponding principal Cauchy stresses arising from the work function (8) then are

$$
\sigma_{r}=\frac{\mu}{V}\left(\lambda_{r}^{2}-1\right)+\lambda(V-1), \quad \sigma_{t}=\frac{\mu}{V}\left(\lambda_{t}^{2}-1\right)+\lambda(V-1), \quad V=\lambda_{r} \lambda_{t}^{2}
$$

The condition of static equilibrium and its boundary conditions are

$$
\frac{d \sigma_{r}}{d r}=\frac{2}{r}\left(\sigma_{t}-\sigma_{r}\right), \quad \sigma_{r} \rightarrow-p_{\infty} \text { as } r \rightarrow \infty, \quad \sigma_{r}=-p_{\text {in }} \text { at } r=a .
$$

These are as given in [1], and it was shown there that for the incompressible limit $(\lambda / \mu \rightarrow \infty)$ the compressed radius $a_{i}$ is determined by

$$
\frac{p_{\infty}-p_{\text {in }}}{\mu}=\frac{1}{2}\left(\frac{a_{0}}{a_{i}}\right)^{4}+2\left(\frac{a_{0}}{a_{i}}\right)-\frac{5}{2} .
$$

We wish to determine the correction to this state when we think of $p_{\infty} / \mu$ and $p_{\text {in }} / \mu$ as fixed and $\epsilon=\mu / \lambda \ll 1$. We suppose that an initial radius $r_{0}$ deforms to $r \sim r_{i}+\epsilon r_{1}+\epsilon^{2} r_{2}+\ldots$, where $r_{i}$ denotes the incompressible solution, and our aim is to calculate the first order correction $r_{1}$. The stretches are

$$
\begin{aligned}
\lambda_{r} & =\frac{d r}{d r_{0}}=r_{i}^{\prime}+\epsilon r_{1}^{\prime}+\epsilon^{2} r_{2}^{\prime}+O\left(\epsilon^{3}\right), \\
\lambda_{t} & =\frac{r}{r_{0}}=\frac{r_{i}}{r_{0}}+\epsilon \frac{r_{1}}{r_{0}}+\epsilon^{2} \frac{r_{2}}{r_{0}}+O\left(\epsilon^{3}\right), \\
V & =\lambda_{r} \lambda_{t}^{2}=V_{0}+\epsilon V_{1}+\epsilon^{2} V_{2}+O\left(\epsilon^{3}\right),
\end{aligned}
$$

where the dashes denote differentiation with respect to $r_{0}$ and

$$
\begin{aligned}
V_{0} & =\frac{r_{i}^{2}}{r_{0}^{2}} r_{i}^{\prime} \\
V_{1} & =\frac{2 r_{i} r_{i}^{\prime} r_{1}}{r_{0}^{2}}+\frac{r_{i}^{2} r_{1}^{\prime}}{r_{0}^{2}}, \\
V_{2} & =\frac{2 r_{i} r_{i}^{\prime} r_{2}}{r_{0}^{2}}+\frac{r_{i}^{\prime} r_{1}^{2}}{r_{0}^{2}}+\frac{2 r_{i} r_{1} r_{1}^{\prime}}{r_{0}^{2}}+\frac{r_{i}^{2} r_{2}^{\prime}}{r_{0}^{2}} .
\end{aligned}
$$

The condition that the stresses remain of order $\mu$ then leads to the incompressibility of the leading order solution, which can be written as

$$
V_{0}=1, \quad r_{i}^{\prime}=\frac{r_{0}^{2}}{r_{i}^{2}}, \quad r_{0}^{3}-r_{i}^{3}=B=a_{0}^{3}-a_{i}^{3} .
$$

The stresses then are

$$
\begin{aligned}
\frac{\sigma_{r}}{\mu} & =\frac{\lambda_{r}^{2}-1}{V}+\frac{V-1}{\epsilon} \\
& =\frac{r_{i}^{\prime 2}-1+2 \epsilon r_{i}^{\prime} r_{1}^{\prime}}{1+\epsilon V_{1}}+V_{1}+\epsilon V_{2}+O\left(\epsilon^{2}\right) \\
& =\left(r_{i}^{\prime 2}-1+V_{1}\right)+\epsilon\left(V_{2}+2 r_{i}^{\prime} r_{1}^{\prime}-\left(r_{i}^{\prime 2}-1\right) V_{1}\right)+O\left(\epsilon^{2}\right), \\
\frac{\sigma_{t}}{\mu} & =\frac{\lambda_{t}^{2}-1}{V}+\frac{V-1}{\epsilon} \\
& =\frac{r_{i}^{2} / r_{0}^{2}-1+2 \epsilon r_{i} r_{1} / r_{0}^{2}}{1+\epsilon V_{1}}+V_{1}+\epsilon V_{2}+O\left(\epsilon^{2}\right) \\
& =\left(r_{i}^{2} / r_{0}^{2}-1+V_{1}\right)+\epsilon\left(V_{2}+2 r_{i} r_{1} / r_{0}^{2}-\left(r_{i}^{2} / r_{0}^{2}-1\right) V_{1}\right)+O\left(\epsilon^{2}\right) .
\end{aligned}
$$


We now substitute these into the condition of static equilibrium (11) and obtain

$$
\begin{aligned}
\frac{1}{\mu} \frac{d \sigma_{r}}{d r_{0}} & =\frac{2}{r} \frac{d r}{d r_{0}}\left(\frac{\sigma_{t}-\sigma_{r}}{\mu}\right) \\
& =\left(\frac{2}{r_{i}}-\epsilon \frac{2 r_{1}}{r_{i}^{2}}\right)\left(r_{i}^{\prime}+\epsilon r_{1}^{\prime}\right)\left\{\Delta+\epsilon\left(\frac{2 r_{i} r_{1}}{r_{0}^{2}}-2 r_{i}^{\prime} r_{1}^{\prime}-\Delta V_{1}\right)\right\}+O\left(\epsilon^{2}\right) \\
& =\frac{2}{r_{i}} r_{i}^{\prime} \Delta+\epsilon L_{1}\left(r_{1}\right)+O\left(\epsilon^{2}\right)
\end{aligned}
$$

where

$$
\Delta=\frac{r_{i}^{2}}{r_{0}^{2}}-r_{i}^{\prime 2}
$$

and $L_{1}$ is the linear operator

$$
L_{1}\left(r_{1}\right)=\left(-2 \frac{r_{1}}{r_{i}^{2}}\right) r_{i}^{\prime} \Delta+\frac{2}{r_{i}} r_{1}^{\prime} \Delta+\frac{2}{r_{i}} r_{i}^{\prime}\left(\frac{2 r_{i} r_{1}}{r_{0}^{2}}-2 r_{i}^{\prime} r_{1}^{\prime}-\Delta V_{1}\right) .
$$

Taking the leading order term in (28) and integrating over $r_{0}$ from $a_{0}$ to infinity we have

$$
\frac{-p_{\infty}+p_{\mathrm{in}}}{\mu}=\int_{a_{0}}^{\infty} \frac{2}{r_{i}} r_{i}^{\prime} \Delta d r_{0}=\int_{a_{0}}^{\infty}\left(\frac{2}{r_{i}}-\frac{2 r_{0}^{6}}{r_{i}^{7}}\right) d r_{0},
$$

where we have used (19b) to eliminate $r_{i}^{\prime}$. There will be other integrals of this form later and we evaluate them using the variable $t=r_{0} / r_{i}>1$ introduced in [1]. In general it gives

$$
\int \frac{r_{0}^{p}}{r_{i}^{q}} d r_{0}=-\int \frac{B^{(p+1-q) / 3} t^{p}}{\left(t^{3}-1\right)^{(p+4-q) / 3}} d t
$$

For (31) then, we obtain

$$
\begin{aligned}
\frac{-p_{\infty}+p_{\text {in }}}{\mu} & =\int_{T}^{1}\left(-\frac{2}{t^{3}-1}+\frac{2 t^{6}}{t^{3}-1}\right) d t \\
& =\int_{T}^{1} 2\left(t^{3}+1\right) d t
\end{aligned}
$$

Thus

$$
\frac{p_{\infty}-p_{\text {in }}}{\mu}=\int_{1}^{T} 2\left(t^{3}+1\right) d t=\frac{1}{2} T^{4}+2 T-\frac{5}{2},
$$

where $T=a_{0} / a_{i}>1$ is the compression ratio of the cavity. This is the result (12) found for the incompressible problem (with this form of $W$ ) in [1].

To proceed from here, we first integrate the leading order term in (28) from a general $r_{0}$ to infinity and obtain

$$
\frac{p_{\infty}+\left(\sigma_{r}\right)_{0}}{\mu}=\frac{1}{2} t^{4}+2 t-\frac{5}{2}
$$

where $\left(\sigma_{r}\right)_{0}$ is the leading order term in $\sigma_{r}$. We therefore have

$$
r_{i}^{\prime 2}-1+V_{1}=\frac{\left(\sigma_{r}\right)_{0}}{\mu}=-\frac{p_{\infty}}{\mu}+\frac{1}{2} t^{4}+2 t-\frac{5}{2} .
$$


But $r_{i}^{\prime}=r_{0}^{2} / r_{i}^{2}=t^{2}$, and $V_{1}$ is given by (17), so we have

$$
\frac{r_{i}^{2}}{r_{0}^{2}} r_{1}^{\prime}+\frac{2 r_{i} r_{i}^{\prime}}{r_{0}^{2}} r_{1}=V_{1}=-\frac{p_{\infty}}{\mu}-\frac{3}{2}-\frac{1}{2} t^{4}+2 t
$$

This we can integrate using an integrating factor and the substitution used earlier, and we obtain

$$
r_{1}=\frac{c r_{0}^{3}}{r_{i}^{2}}+\frac{r_{0}^{4}}{2 r_{i}^{3}}+\frac{A}{r_{i}^{2}},
$$

where $A$ is an arbitrary constant and $c=-\frac{1}{2}-p_{\infty} /(3 \mu)$. It is the need to determine this constant $A$ that forces us to consider the next terms in the equation. In fact, since we have exactly matched $\sigma_{r} / \mu$ to its boundary values at leading order, when we integrate (28) from $a_{0}$ to infinity we must have

$$
\int_{a_{0}}^{\infty} L_{1}\left(r_{1}\right) d r_{0}=0
$$

and this is the linear equation that will determine $A$. In detail, when we substitute (17) into $L_{1}$ we obtain

$$
L_{1}\left(r_{1}\right)=-\frac{2 r_{1}}{r_{i}^{2}}+\frac{6 r_{0}^{6} r_{1}}{r_{i}^{8}}-\frac{4 r_{0}^{4} r_{1}^{\prime}}{r_{i}^{5}}
$$

where we have used (19b) again to eliminate $r_{i}^{\prime}$. Then when we substitute (39) into this we obtain

$$
L_{1}\left(r_{1}\right)=-\frac{2 c r_{0}^{3}}{r_{i}^{4}}-\frac{r_{0}^{4}}{r_{i}^{5}}-\frac{12 c r_{0}^{6}}{r_{i}^{7}}-\frac{8 r_{0}^{7}}{r_{i}^{8}}+\frac{14 c r_{0}^{9}}{r_{i}^{10}}+\frac{9 r_{0}^{10}}{r_{i}^{11}}-\frac{2 A}{r_{i}^{4}}+\frac{14 A r_{0}^{6}}{r_{i}^{10}}
$$

Then writing down (40), using the $t$-substitution and (32), we obtain

$$
\begin{aligned}
0 & =\int_{T}^{1}\left(\frac{2 c t^{3}+t^{4}+12 c t^{6}+8 t^{7}-14 c t^{9}-9 t^{10}}{t^{3}-1}+\frac{2 A}{B}-\frac{14 A t^{6}}{B}\right) d t \\
& =\int_{T}^{1}-2 c\left(t^{3}+7 t^{6}\right)-\left(t^{4}+9 t^{7}\right)+\frac{2 A}{B}\left(1-7 t^{6}\right) d t \\
& =\left[-2 c\left(\frac{t^{4}}{4}+t^{7}\right)-\left(\frac{9 t^{8}}{8}+\frac{t^{5}}{5}\right)+\frac{2 A}{B}\left(t-t^{7}\right)\right]_{T}^{1} \\
& =2 c\left(T^{7}+\frac{T^{4}}{4}-\frac{5}{4}\right)+\left(\frac{9 T^{8}}{8}+\frac{T^{5}}{5}-\frac{53}{40}\right)+\frac{2 A}{B}\left(T^{7}-T\right) .
\end{aligned}
$$

In the last term we can write $\left(T^{3}-1\right) / B=1 / a_{i}^{3}$, so the last term is $2 A\left(T^{4}+T\right) / a_{i}^{3}$, and finally we obtain

$$
A=-\frac{a_{i}^{3}}{T^{4}+T}\left\{c\left(T^{7}+\frac{T^{4}}{4}-\frac{5}{4}\right)+\left(\frac{9 T^{8}}{16}+\frac{T^{5}}{10}-\frac{53}{80}\right)\right\} .
$$

The resulting curves, plotted in Figure 3, are in good agreement with a fully nonlinear elastostatic calculation (carried out by Andrew Hazel). 


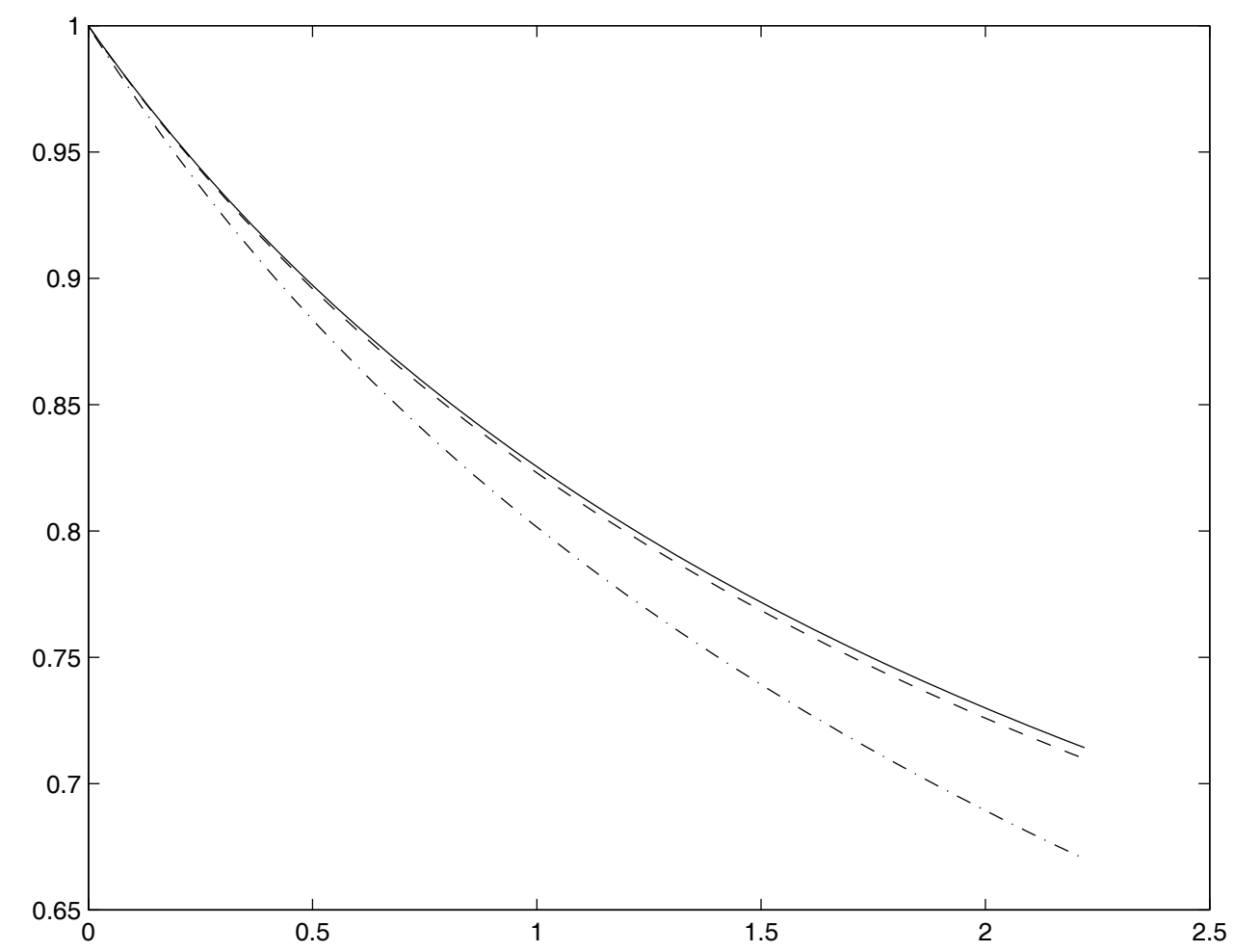

Figure 1: Compression ratio $a / a_{0}$ as a function of $p_{\infty} / \mu$ with $p_{\text {in }} / \mu=0$, for $\epsilon=0$ (solid), $\epsilon=10^{-2}$ (dashed) and $\epsilon=10^{-1}$ (dot-dashed).

\section{Scattering from the strained region}

The problem of acoustic scattering from a strained region may be solved via the technique of small displacements superposed on the finite deformation (otherwise known as "small on large" theory), using the stressed deformation as the reference configuration for the scattering problem, illustrated in figure 4 .

For our problem the specific configuration is shown in figure 4 , so that the cavity of initial radius $a_{0}$ in the configuration $B_{0}$ is finitely deformed via a pressure $p_{\infty}$ at infinity to a radius $a$ in the configuration $B$. For the purposes of the study group problem we are mainly interested in the monopole scattering characteristics since this is the dominant contribution to the scattering cross section of the cavity.

The section is divided into two parts. Firstly we analyse the problem formally by appealing to the "small on large" theory. However the complexity of this approach motivates a physical argument which leads to a more direct estimate of the monopole scattering cross section. 


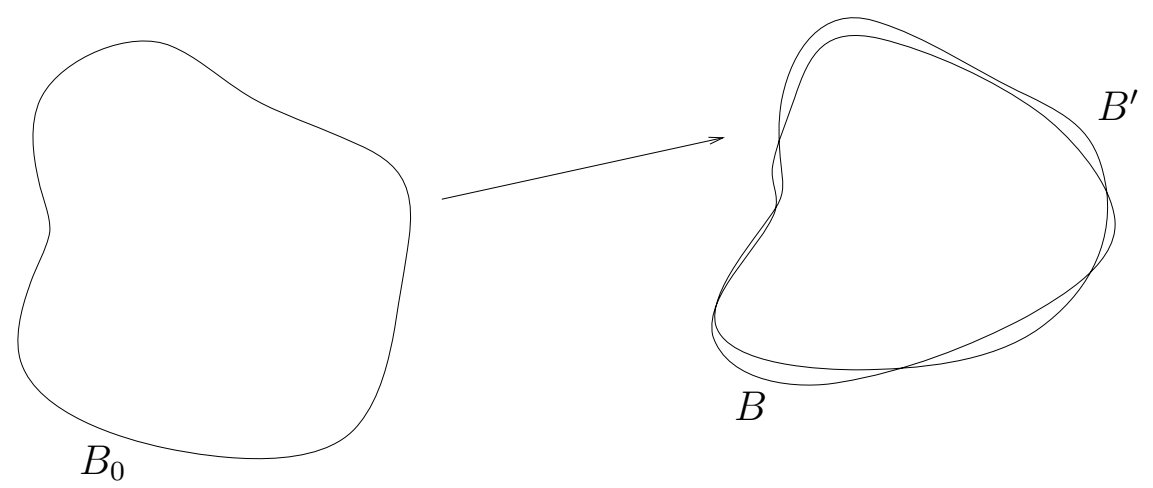

Figure 2: Figure to show the initial finite deformation from $B_{0}$ to $B$ and the small displacements superposed on $B$, deforming the material into the configuration $B^{\prime}$.

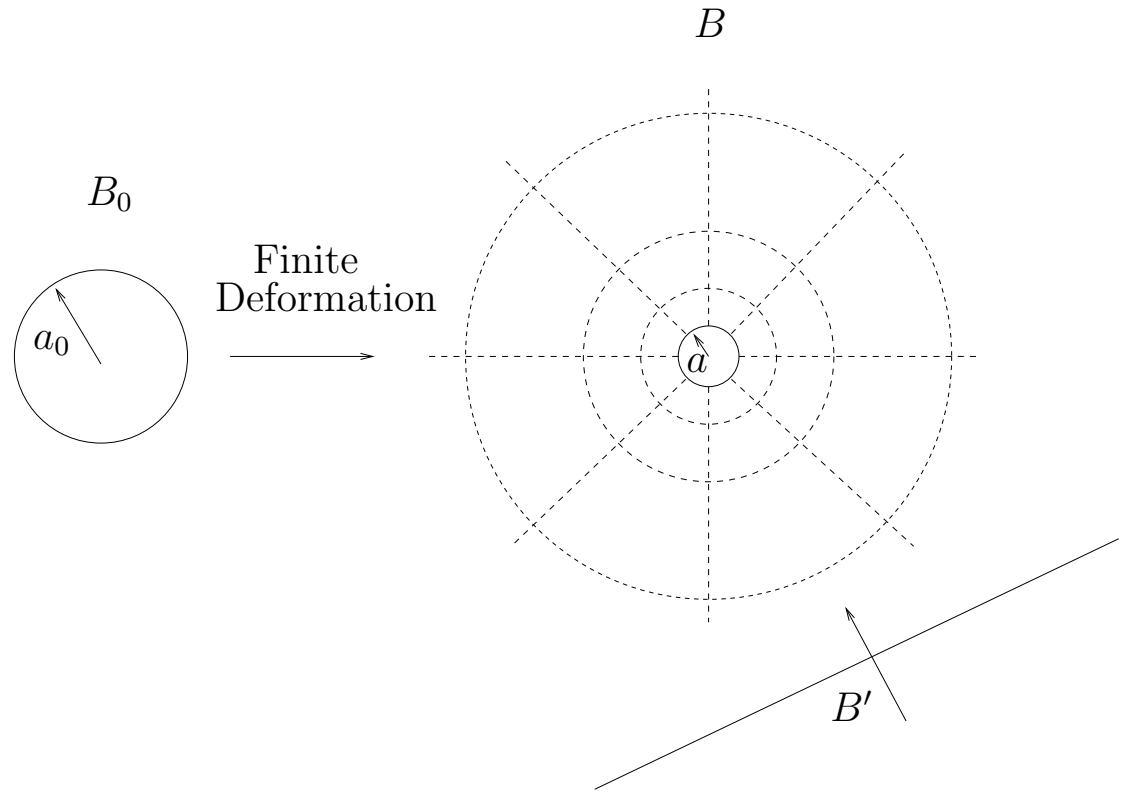

Figure 3: The configuration of the problem of scattering from a strained region around a spherical cavity. $B_{0}$ is the configuration of the undeformed material. $B$ is the configuration of the medium once the finite deformation has taken place (straining is represented by the dashed lines) and finally $B^{\prime}$ is the configuration of the strained medium with additional small deformations corresponding to the wave field.

\subsection{Formal Analysis of the Scattering Problem}

We will take $B$ as the reference state about which we linearize to find the equation of motion for the additional small displacements which correspond to the incident (and scattered) acoustic waves. 
We may use the "small on large" theory of Green and Zerna [4], (as was done in [1] for an initial incompressible finite deformation), in order to derive the full linear wave equations governing the scattered displacement field $\left(u_{r}, u_{\theta}\right)$ with the condition of axisymmetry:

$$
\begin{aligned}
A(r) \frac{\partial^{2} u_{r}}{\partial r^{2}}+B(r) \frac{1}{r^{2}} \frac{\partial^{2} u_{r}}{\partial \theta^{2}}+ & C(r) \frac{1}{r} \frac{\partial^{2} u_{\theta}}{\partial r \partial \theta}+\ldots \\
& +J(r) \frac{1}{r^{2}} u_{r}+K(r) \frac{\cot \theta}{r^{2}} u_{\theta}-\rho \frac{\partial^{2} u_{r}}{\partial t^{2}}=-F_{r}\left(r, \theta, \mathbf{u}_{\text {inc }}\right) \\
L(r) \frac{\partial^{2} u_{\theta}}{\partial r^{2}}+M(r) \frac{1}{r^{2}} \frac{\partial^{2} u_{\theta}}{\partial \theta^{2}}+ & N(r) \frac{1}{r} \frac{\partial^{2} u_{r}}{\partial r \partial \theta}+\ldots \\
& +U(r) \frac{1}{r^{2}} u_{r}+V(r) \frac{\csc ^{2} \theta}{r^{2}} u_{\theta}-\rho \frac{\partial^{2} u_{\theta}}{\partial t^{2}}=-F_{\theta}\left(r, \theta, \mathbf{u}_{\text {inc }}\right)
\end{aligned}
$$

where $A(r), B(r), \ldots, V(r)$ are coefficients which depend on the elastic constants of the material and the initial finite deformation (hence the $r$ dependence). The terms $F_{r}$ and $F_{\theta}$ are the forcing terms resulting from the differential operators on the left hand side of the equations operating on the incident displacement field $\mathbf{u}_{\text {inc }}$.

For the study group problem we were asked to try to understand the monopole scattering behaviour (due to an incident P-wave), since this was thought to be by far the strongest. The part of the scattered field associated with the monopole scattering will depend on $r$ only (and $t$ ) and since for axisymmetric problems $u_{\theta}$ cannot be a function only of $r$ its monopole contribution must be zero. Thus we can write the scattered displacement in the form

$$
\begin{aligned}
& u_{r}(r, \theta, t)=U(r, t)+\text { higher order scattering, } \\
& u_{\theta}(r, \theta, t)=0+\text { higher order scattering, }
\end{aligned}
$$

where $U(r, t)$ is associated with the monopole scattering. Thus we are looking for that part of the solution of (48) and (49) which depends on $r$ (and $t$ ) only.

If we consider an incident plane wave of the form

$$
\mathbf{u}_{\mathrm{inc}}=u_{1} \mathbf{e}_{z} \exp i(\omega t-k z),
$$

then the stresses in this are

$$
\left(\sigma_{m n}^{\mathrm{inc}}\right)=\left(\begin{array}{ccc}
-\lambda^{\prime} i k u_{1} & 0 & 0 \\
0 & -\lambda^{\prime} i k u_{1} & 0 \\
0 & 0 & -\left(\lambda^{\prime}+2 \mu\right) i k u_{1}
\end{array}\right) e^{i(\omega t-k z)},
$$

where $\lambda^{\prime}$ is the effective Lamé modulus of the material modified by the static pressure $p_{\infty}$. (This is in fact $\lambda^{\prime}=\lambda-p_{\infty}+O(\epsilon \mu)$ when $p_{\infty}=O(\mu)$ as we consider.) Since $\lambda^{\prime} \gg \mu$, this stress tensor is, at leading order, just isotropic,

$$
\sigma_{m n}^{\text {inc }}=\left(-\lambda i k u_{1}+O(\mu)\right) \delta_{m n} e^{i(\omega t-k z)} .
$$

As we are interested in the low frequency regime $(a \ll \lambda)$ we have $k a \ll 1$, and so $\exp (-i k z)$ can be approximated by 1 in the scattering region. (We note that for $p_{\infty}$ of 
order $\mu$, the size of the region where significant strain occurs around the microsphere is of order a.) We therefore approximate the incident forcing responsible for monopole scattering as

$$
\sigma_{r r}^{\mathrm{inc}}=-\lambda i k u_{1} e^{i \omega t}
$$

If we then write the radial displacement in the monopole scattered field as

$$
U(r, t)=u_{1} U(r) e^{i \omega t}
$$

we find that

$$
A(r) \frac{\partial^{2} U}{\partial r^{2}}+D(r) \frac{1}{r} \frac{\partial U}{\partial r}+\left(J(r) \frac{1}{r^{2}}+\rho \omega^{2}\right) U=-\left(J(r) \frac{1}{r^{2}}+\rho \omega^{2}\right) .
$$

Here $U(r)$ is a scaled displacement of course, entirely distinct from the elastic coefficient $U(r)$ in (49). In order to determine the coefficients $A(r), D(r)$ and $J(r)$ we should use the solution of the slightly compressible finite deformation problem found above. It is clear that the coefficients $A(r), D(r)$ and $J(r)$ should then tend to those of the linear Navier equations with an additional pressure of that imposed at infinity (and hence a pre-stressed substrate). Thus as $r \rightarrow \infty$ we should find that

$$
\begin{array}{r}
A(r) \rightarrow 2 \mu+\lambda-p_{\infty}, \\
D(r) \rightarrow 2\left(2 \mu+\lambda-p_{\infty}\right), \\
J(r) \rightarrow-2\left(2 \mu+\lambda-p_{\infty}\right) .
\end{array}
$$

These coefficients can be found as a function of the principal stretches given in equations (13) and (14) above and using the approach in [1] and [4]. Unfortunately due to time constraints these coefficients were not found during the study group but certain contributors hope to address this problem shortly.

Note also that the shear modulus appearing in these coefficients is the dynamic shear modulus and referring to the discussion of constitutive behaviour above, it is reasonable to directly replace the static shear modulus $\mu$ by the dynamic shear modulus $\mu(\omega)$ for a single cavity in an infinite medium.

Once the coefficients $A(r), D(r)$ and $J(r)$ have been found, (57) can be solved either numerically or via asymptotic matching in order to find the scattered field $U(r, t)$ and hence the monopole scattering coefficient is obtained:

$$
\gamma_{\text {monopole }}=\frac{\text { energy scattered (via monopole) per unit time }}{\text { energy per unit area carried by incident wave per unit time }}
$$

where for the monopole contribution the rate at which energy is being scattered is

$$
\iint \Sigma_{r r} \frac{\partial U}{\partial t} d S
$$

where $S$ is some spherical surface concentric with the cavity, having a radius greater than that of the cavity, and $\Sigma_{r r}$ is the additional stress due to the monopole scattered field. 


\subsection{Physical Arguments}

Instead of the formal approach used in the previous section, we may repeat the physical argument given in [1] but now use the slightly compressible initial deformation and the dynamic shear modulus $\mu(\omega)$.

With reference to figure 4.2, we suppose that all the stress induced anisotropy (the strained region) is located in a region local to the cavity, characterised by a lengthscale $L$ which although much greater than the deformed radius $a$ of the cavity is still much less than the wavelength of the incident wave $\lambda$. We suppose that the exterior of this region remains isotropic.

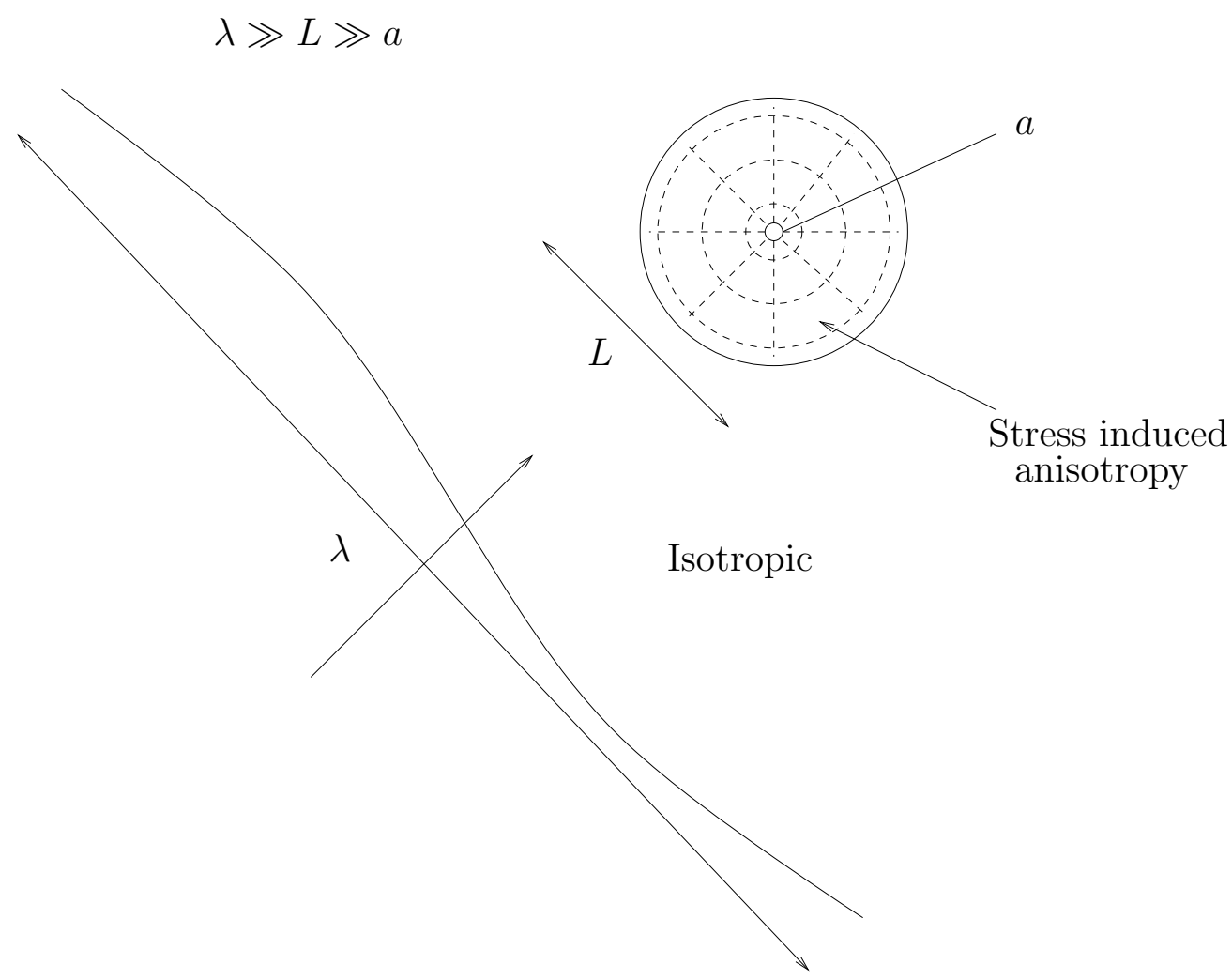

Figure 4: Illustration of the geometry of the problem and the lengthscales involved in the physical argument.

It is clear that monopole behaviour relates to volume changes. On considering an incident field of the form (52-53) the cavity will see (at leading order) the pressure field

$$
p=p_{s}+p_{1} \cos (\omega t-k z)
$$

where $p_{s}$ is the steady static pressure and $p_{1}$ is the acoustic amplitude. Referring once more to figure 4.2, for low frequencies the inner region will be subjected to a slowly varying static pressure and since $\lambda \gg L$ it will respond quasi-statically, since to leading order it will see only the pressure field

$$
p=p_{s}+p_{1} \cos (\omega t) .
$$


Thus the radius of the compressed cavity varies according to:

$$
a=a_{s}+a_{1} \cos (\omega t)
$$

where $a_{s}$ is the compressed radius of the micro-sphere under the static pressure $p_{s}$ and

$$
a_{1}=\left.\left(\frac{d a}{d p}\right)\right|_{p_{s}} \frac{1}{3} p_{1}
$$

which is small and therefore the volume fluctuation is approximately $V(t) \approx$ $4 \pi a_{s}^{2} a_{1} \cos (\omega t)$.

Since the outer region is approximately isotropic it responds like an acoustic medium to the volume fluctuation and thus the radiation of energy is (Lighthill [5])

$$
E_{R}=\frac{\rho(\ddot{V})^{2}}{4 \pi c_{p}}
$$

Now define

$$
E_{S}=\int_{0}^{2 \pi / \omega} E_{R} d t=\frac{2 \pi \rho a_{s}^{4} \omega^{4} p_{1}^{2}}{c_{p}}\left(\left.\left(\frac{d a}{d p}\right)\right|_{p_{s}}\right)^{2}
$$

which is the time averaged radiated energy.

The incident energy flux density is

$$
E_{I}=\frac{p_{1}^{2}}{2 \rho c_{p}}
$$

and thus

$$
\gamma_{\text {monopole }} \sim \frac{4 \pi^{2}}{\rho} a_{s}^{4} \omega^{4}\left(\left.\left(\frac{d a}{d p}\right)\right|_{p_{s}}\right)^{2}
$$

In [1], both $a_{s}$ and $d a /\left.d p\right|_{p_{s}}$ were calculated via the incompressible solution of the static problem and with the static shear modulus $\mu$. They may now be calculated via the small compressibility static solution obtained above. Furthermore we may directly replace $\mu$ by $\mu(\omega)$, the dynamic shear modulus for scattering by a single spherical cavity.

\section{Conclusions}

In this study group report we have considered the problem of acoustic scattering from a strained region where the shear modulus is considered frequency dependent. We argued that since the material exterior to the strained region is homogeneous we may simply replace the static shear modulus with the dynamic one given in (5). Different models 
are, however, needed for filled rubbers and this could be significant in "real" materials where we may not be able to neglect interaction effects between inclusions (cavities).

The initial static problem (originally considered in [1]) was extended in order to include small compressibility, a notion essential so that compressive waves can travel through the material. The "next order" terms were found for the deformed radius and we compared values of the compression ratio $a / a_{0}$ for a range of $p_{\infty} / \mu$ obtained via $\epsilon=0$ (the incompressible case) with various compressible solutions with $\epsilon \ll 1$.

We considered two approaches to the scattering problem. The first is the formal approach using the "small on large" theory. Restricting attention to monopole scattering enabled us to consider a simplified (although still complicated!) equation (57), the coefficients of which depend on the (corrected) static problem allowing for small compressibility. The complexity of this problem motivated a physical argument in order to deduce an expression for the monopole scattering coefficient which was given in (70). This expression is a function of $a_{s}$ and $d a /\left.d p\right|_{p_{s}}$ which should now be calculated via the solution to the slightly compressible static deformation solved above (with dynamic shear modulus) rather than using the incompressible solution obtained in [1].

\section{References}

[1] Elastic scattering from a strained region. MSc dissertation of W.J.Parnell. http://eprints.maths.ox.ac.uk/archive/00000014/.

[2] On a fully three-dimensional finite-strain viscoelastic damage model: formulation and computational aspects. J.C.Simo. Computer methods in applied mechanics and engineering 60, 153-173, 1987.

[3] A constitutive model and FEA of rubber under small oscillatory load superimposed on large static deformation. B.-K. Kim, S.-K. Youn, and W.-S. Lee. Archive of applied mechanics 73, 781-798, 2004.

[4] Theoretical elasticity. A.E.Green and W.Zerna. 2nd edition, OUP, 1968.

[5] Waves in fluids. James Lighthill. CUP, 1978. 


\title{
Acoustic scattering from a strained region
}

\author{
Problem presented by \\ Peter Brazier-Smith \\ Thales Underwater Systems
}

\section{Problem statement}

A composite material consists of a rubber filled with gas-filled microspheres. In underwater applications it is compressed hydrostatically by a pressure that may be not insignificant compared with the shear modulus of the rubber, so large strains are produced around each spherical inclusion. When these spherical inclusions scatter an incident acoustic wave, the strained region around an inclusion has had its elastic properties altered by the large static strain, and the Study Group was asked to address the question of how this strained region affects the elastic scattering, bearing in mind that the dynamic shear modulus differs from its static value. The acoustic wavelength is large compared with the diameter of the spherical inclusions and, for the purposes of the Study Group, scattering by just a single microsphere was considered. The Study Group showed that for the monopole scattering strength it was enough to use the dynamic shear modulus in place of the static value in the existing approach, and also obtained the equations that would have to be tackled for finding the dipole and other scattering strengths.

\section{Study Group contributors}

\author{
David Abrahams (University of Manchester) \\ David Allwright (Smith Institute) \\ Chris Bell (University of Oxford) \\ Tamas Bodai (University of Aberdeen) \\ Philip Bond (University of Oxford) \\ Andrew Hazel (University of Manchester) \\ Gareth Jones (University of Oxford) \\ Roman Novokshanov (University of Oxford) \\ David Parker (University of Edinburgh) \\ William Parnell (University of Manchester) \\ Emmanuel Perrey-Debain (University of Manchester) \\ Ben Veitch (University of Manchester)
}




\title{
Report prepared by
}

\author{
David Allwright (Smith Institute) \\ Gareth Jones (University of Oxford) \\ William Parnell (University of Manchester)
}

\section{Introduction}

A composite material consists of a rubber filled with gas-filled microspheres. In underwater applications it is compressed hydrostatically by a pressure that may be not insignificant compared with the shear modulus of the rubber. Thus large strains are produced around each spherical inclusion. When these spherical inclusions scatter an incident acoustic wave, the strained region around an inclusion has had its elastic properties altered by the large static strain. How does this strained region affect the elastic scattering? The acoustic wavelength is large compared with the diameter of the spherical inclusions. For the purposes of this Study Group problem, the homogenization problem (obtaining the macroscopic behaviour of the bulk material from the behaviour of a single microsphere) is to be assumed solvable. So the Study Group is asked to focus on scattering by a single microsphere and the strained region around it. The particular concern that the Study Group is asked to focus on is that the material dynamic moduli are different from the static moduli, and we wish to allow properly for this in the analysis. The bulk modulus can be treated as constant, but the dynamic shear modulus of the rubber can increase by at least an order of magnitude over its static value at frequencies of interest. A typical variation with frequency at 2 different temperatures is shown below. Previous work on this problem by W.J.Parnell is in [1].

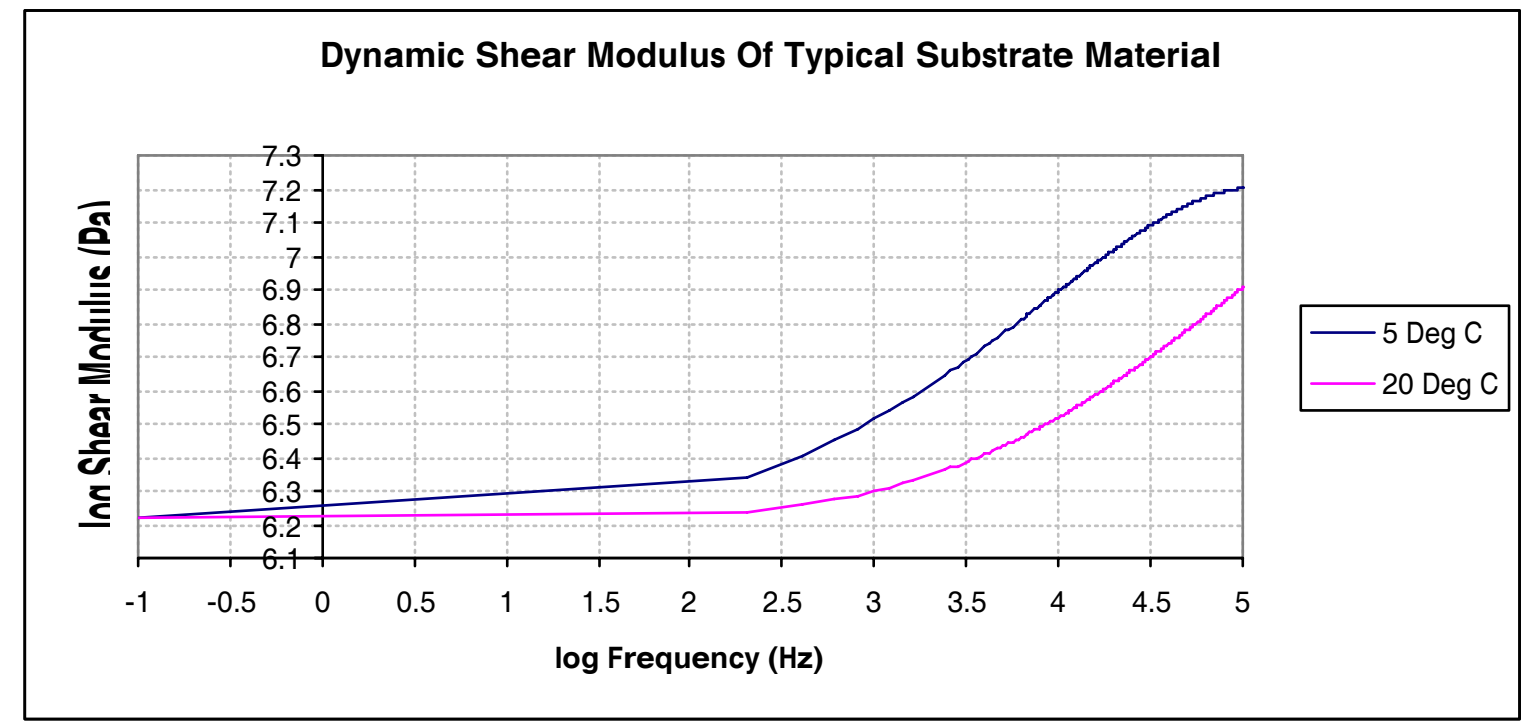

In this problem report, the work is divided up into various sections. First, there are obviously some material modelling issues: a mathematical model is needed for small 
strain dynamic viscoelasticity superimposed on a large static strain. Some of the existing models for this are reviewed in Section 2. Then, in order to formulate the scattering problem for compressive waves correctly, we need to compute the static state allowing for a finite compressibility. This is in contrast to [1], where the static state was only computed explicitly for an incompressible material, and then this was used as the basis for an approximate computation of the monopole scattering cross-section. Our calculation of the correction to this static state, to allow for finite compressibility, is in Section 3. Then in Section 4 we address the scattering problem itself.

\section{$2 \quad$ Material Modelling}

There are in the literature a number of models for dynamic large strain viscoelasticity of rubbery materials, and in particular we referred to papers by Simo [2] and by Kim, Youn and Lee [3].

Simo's model initially assumes a single time-constant $\nu$ in the viscoelastic behaviour, but this is generalized later. We initially treat the unstressed state of the rubber as the reference configuration, and let the deformation gradient matrix at any point be $F$, so $J=\operatorname{det}(F)$ is the volume ratio. The deviatoric part of the deformation is written as

$$
\bar{E}=\frac{1}{2}\left(\frac{F^{T} F}{J^{2 / 3}}-I\right)
$$

so this represents the change of shape of a material element. Then the free energy is written in the form

$$
U^{0}(J)+\bar{\Psi}_{0}(\bar{E})-Q: \bar{E}+\Psi_{I}(Q) .
$$

Here, $U^{0}(J)$ represents the term due to change of volume, and if $K_{0}$ is the bulk modulus then the correct form for small deformations is

$$
U^{0}(J)=\frac{1}{2} K_{0}(J-1)^{2} .
$$

The matrix $Q$ is an internal variable representing the relaxation process, and will be governed by a first order equation in time

$$
\frac{d Q}{d t}+\frac{Q}{\nu}=\frac{1-\gamma}{\nu} \operatorname{DEV}\left(\frac{\partial \bar{\Psi}_{0}(\bar{E})}{\partial \bar{E}}\right)
$$

where $\operatorname{DEV}($.) denotes taking the deviatoric part. In this, $0<\gamma<1$ and of course $\nu>0$.

This model has the following features:

(1) By choice of $\bar{\Psi}_{0}$ it can be matched to a given measured static large-strain behaviour.

(2) For small dynamic strains about the unstressed state, it gives a dynamic shear modulus

$$
\mu(\omega)=\mu^{0}\left(\frac{\gamma+i \omega \nu}{1+i \omega \nu}\right)
$$


where $\gamma \mu^{0}$ and $\mu^{0}$ are the rubbery and glassy shear modulus (i.e. the low frequency and high frequency limits). The transition region between these limits is where $\omega \nu=O(1)$.

(3) The model agrees well with experiments on pure rubbers. (Different models, developed in [3], are needed for "filled" rubbers, i.e. rubbers filled with, for instance, carbon particles.)

(4) The model predicts that the dynamic moduli for small deviations about a large static strain are got by taking the corresponding static moduli and replacing the static shear modulus $\mu$ by $\mu(\omega)$. For instance, suppose the (1111) static differential stiffness is $\mu \lambda_{1}$, where $\lambda_{1}$ is a principal stretch, so small static increments to strain and stress obey

$$
\delta \sigma_{11}=\mu \lambda_{1} \delta e_{11}+\ldots
$$

Then in a dynamic problem at frequency $\omega$, with $\delta e_{i j}=\Re\left(\delta \tilde{e}_{i j} e^{i \omega t}\right)$ and $\delta \sigma_{i j}=$ $\Re\left(\delta \tilde{\sigma}_{i j} e^{i \omega t}\right)$, we shall have

$$
\delta \tilde{\sigma}_{11}=\mu(\omega) \lambda_{1} \delta \tilde{e}_{11}+\ldots
$$

In other words, $\mu(\omega) \lambda_{1}$ is the nonlinear dynamic stiffness.

(5) Viscoelastic models with multiple relaxation times can be handled in the same way, and the upshot is that one replaces the factor $\mu$ in the static incremental moduli by $\mu(\omega)$ to obtain the dynamic incremental modulus. The values of this shear modulus can either be measured directly, or inferred from a measured temperaturedependence by the WLF rule in ways that are well-known.

\section{Static state: compressibility correction}

To analyse compressive waves as departures from a strained state, we must start from a strained state that allows for a finite compressibility. We therefore consider a spherical cavity of initial radius $a_{0}$, in a material with static strain energy function

$$
W=\frac{1}{2} \mu\left(\lambda_{1}^{2}+\lambda_{2}^{2}+\lambda_{3}^{2}-3-2 \log V\right)+\frac{1}{2} \lambda(V-1)^{2} .
$$

In this, $\lambda$ and $\mu$ are the usual Lamé moduli, the $\lambda_{i}$ are the principal stretches of a material element, and $V=\lambda_{1} \lambda_{2} \lambda_{3}$ is the volume ratio. This is referred to as a compressible neoHookean material. We assume that the compressed state has a uniform hydrostatic pressure $p_{\infty}$ as $r \rightarrow \infty$, a pressure $p_{\text {in }}$ in the cavity, and a compressed radius $a$. The whole deformation is spherically symmetric, so the material at a given radius $r_{0}>a_{0}$ in the unstressed state will deform to some radius $r>a$ in the stressed state, and the principal stretches $\lambda_{r}$ radially and $\lambda_{t}$ tangentially will be

$$
\lambda_{r}=\frac{d r}{d r_{0}}, \quad \lambda_{t}=\frac{r}{r_{0}} .
$$


The corresponding principal Cauchy stresses arising from the work function (8) then are

$$
\sigma_{r}=\frac{\mu}{V}\left(\lambda_{r}^{2}-1\right)+\lambda(V-1), \quad \sigma_{t}=\frac{\mu}{V}\left(\lambda_{t}^{2}-1\right)+\lambda(V-1), \quad V=\lambda_{r} \lambda_{t}^{2}
$$

The condition of static equilibrium and its boundary conditions are

$$
\frac{d \sigma_{r}}{d r}=\frac{2}{r}\left(\sigma_{t}-\sigma_{r}\right), \quad \sigma_{r} \rightarrow-p_{\infty} \text { as } r \rightarrow \infty, \quad \sigma_{r}=-p_{\text {in }} \text { at } r=a .
$$

These are as given in [1], and it was shown there that for the incompressible limit $(\lambda / \mu \rightarrow \infty)$ the compressed radius $a_{i}$ is determined by

$$
\frac{p_{\infty}-p_{\text {in }}}{\mu}=\frac{1}{2}\left(\frac{a_{0}}{a_{i}}\right)^{4}+2\left(\frac{a_{0}}{a_{i}}\right)-\frac{5}{2} .
$$

We wish to determine the correction to this state when we think of $p_{\infty} / \mu$ and $p_{\text {in }} / \mu$ as fixed and $\epsilon=\mu / \lambda \ll 1$. We suppose that an initial radius $r_{0}$ deforms to $r \sim r_{i}+\epsilon r_{1}+\epsilon^{2} r_{2}+\ldots$, where $r_{i}$ denotes the incompressible solution, and our aim is to calculate the first order correction $r_{1}$. The stretches are

$$
\begin{aligned}
\lambda_{r} & =\frac{d r}{d r_{0}}=r_{i}^{\prime}+\epsilon r_{1}^{\prime}+\epsilon^{2} r_{2}^{\prime}+O\left(\epsilon^{3}\right), \\
\lambda_{t} & =\frac{r}{r_{0}}=\frac{r_{i}}{r_{0}}+\epsilon \frac{r_{1}}{r_{0}}+\epsilon^{2} \frac{r_{2}}{r_{0}}+O\left(\epsilon^{3}\right), \\
V & =\lambda_{r} \lambda_{t}^{2}=V_{0}+\epsilon V_{1}+\epsilon^{2} V_{2}+O\left(\epsilon^{3}\right),
\end{aligned}
$$

where the dashes denote differentiation with respect to $r_{0}$ and

$$
\begin{aligned}
V_{0} & =\frac{r_{i}^{2}}{r_{0}^{2}} r_{i}^{\prime} \\
V_{1} & =\frac{2 r_{i} r_{i}^{\prime} r_{1}}{r_{0}^{2}}+\frac{r_{i}^{2} r_{1}^{\prime}}{r_{0}^{2}}, \\
V_{2} & =\frac{2 r_{i} r_{i}^{\prime} r_{2}}{r_{0}^{2}}+\frac{r_{i}^{\prime} r_{1}^{2}}{r_{0}^{2}}+\frac{2 r_{i} r_{1} r_{1}^{\prime}}{r_{0}^{2}}+\frac{r_{i}^{2} r_{2}^{\prime}}{r_{0}^{2}} .
\end{aligned}
$$

The condition that the stresses remain of order $\mu$ then leads to the incompressibility of the leading order solution, which can be written as

$$
V_{0}=1, \quad r_{i}^{\prime}=\frac{r_{0}^{2}}{r_{i}^{2}}, \quad r_{0}^{3}-r_{i}^{3}=B=a_{0}^{3}-a_{i}^{3} .
$$

The stresses then are

$$
\begin{aligned}
\frac{\sigma_{r}}{\mu} & =\frac{\lambda_{r}^{2}-1}{V}+\frac{V-1}{\epsilon} \\
& =\frac{r_{i}^{\prime 2}-1+2 \epsilon r_{i}^{\prime} r_{1}^{\prime}}{1+\epsilon V_{1}}+V_{1}+\epsilon V_{2}+O\left(\epsilon^{2}\right) \\
& =\left(r_{i}^{\prime 2}-1+V_{1}\right)+\epsilon\left(V_{2}+2 r_{i}^{\prime} r_{1}^{\prime}-\left(r_{i}^{\prime 2}-1\right) V_{1}\right)+O\left(\epsilon^{2}\right), \\
\frac{\sigma_{t}}{\mu} & =\frac{\lambda_{t}^{2}-1}{V}+\frac{V-1}{\epsilon} \\
& =\frac{r_{i}^{2} / r_{0}^{2}-1+2 \epsilon r_{i} r_{1} / r_{0}^{2}}{1+\epsilon V_{1}}+V_{1}+\epsilon V_{2}+O\left(\epsilon^{2}\right) \\
& =\left(r_{i}^{2} / r_{0}^{2}-1+V_{1}\right)+\epsilon\left(V_{2}+2 r_{i} r_{1} / r_{0}^{2}-\left(r_{i}^{2} / r_{0}^{2}-1\right) V_{1}\right)+O\left(\epsilon^{2}\right) .
\end{aligned}
$$


We now substitute these into the condition of static equilibrium (11) and obtain

$$
\begin{aligned}
\frac{1}{\mu} \frac{d \sigma_{r}}{d r_{0}} & =\frac{2}{r} \frac{d r}{d r_{0}}\left(\frac{\sigma_{t}-\sigma_{r}}{\mu}\right) \\
& =\left(\frac{2}{r_{i}}-\epsilon \frac{2 r_{1}}{r_{i}^{2}}\right)\left(r_{i}^{\prime}+\epsilon r_{1}^{\prime}\right)\left\{\Delta+\epsilon\left(\frac{2 r_{i} r_{1}}{r_{0}^{2}}-2 r_{i}^{\prime} r_{1}^{\prime}-\Delta V_{1}\right)\right\}+O\left(\epsilon^{2}\right) \\
& =\frac{2}{r_{i}} r_{i}^{\prime} \Delta+\epsilon L_{1}\left(r_{1}\right)+O\left(\epsilon^{2}\right)
\end{aligned}
$$

where

$$
\Delta=\frac{r_{i}^{2}}{r_{0}^{2}}-r_{i}^{\prime 2}
$$

and $L_{1}$ is the linear operator

$$
L_{1}\left(r_{1}\right)=\left(-2 \frac{r_{1}}{r_{i}^{2}}\right) r_{i}^{\prime} \Delta+\frac{2}{r_{i}} r_{1}^{\prime} \Delta+\frac{2}{r_{i}} r_{i}^{\prime}\left(\frac{2 r_{i} r_{1}}{r_{0}^{2}}-2 r_{i}^{\prime} r_{1}^{\prime}-\Delta V_{1}\right) .
$$

Taking the leading order term in (28) and integrating over $r_{0}$ from $a_{0}$ to infinity we have

$$
\frac{-p_{\infty}+p_{\mathrm{in}}}{\mu}=\int_{a_{0}}^{\infty} \frac{2}{r_{i}} r_{i}^{\prime} \Delta d r_{0}=\int_{a_{0}}^{\infty}\left(\frac{2}{r_{i}}-\frac{2 r_{0}^{6}}{r_{i}^{7}}\right) d r_{0},
$$

where we have used (19b) to eliminate $r_{i}^{\prime}$. There will be other integrals of this form later and we evaluate them using the variable $t=r_{0} / r_{i}>1$ introduced in [1]. In general it gives

$$
\int \frac{r_{0}^{p}}{r_{i}^{q}} d r_{0}=-\int \frac{B^{(p+1-q) / 3} t^{p}}{\left(t^{3}-1\right)^{(p+4-q) / 3}} d t
$$

For (31) then, we obtain

$$
\begin{aligned}
\frac{-p_{\infty}+p_{\text {in }}}{\mu} & =\int_{T}^{1}\left(-\frac{2}{t^{3}-1}+\frac{2 t^{6}}{t^{3}-1}\right) d t \\
& =\int_{T}^{1} 2\left(t^{3}+1\right) d t
\end{aligned}
$$

Thus

$$
\frac{p_{\infty}-p_{\text {in }}}{\mu}=\int_{1}^{T} 2\left(t^{3}+1\right) d t=\frac{1}{2} T^{4}+2 T-\frac{5}{2},
$$

where $T=a_{0} / a_{i}>1$ is the compression ratio of the cavity. This is the result (12) found for the incompressible problem (with this form of $W$ ) in [1].

To proceed from here, we first integrate the leading order term in (28) from a general $r_{0}$ to infinity and obtain

$$
\frac{p_{\infty}+\left(\sigma_{r}\right)_{0}}{\mu}=\frac{1}{2} t^{4}+2 t-\frac{5}{2}
$$

where $\left(\sigma_{r}\right)_{0}$ is the leading order term in $\sigma_{r}$. We therefore have

$$
r_{i}^{\prime 2}-1+V_{1}=\frac{\left(\sigma_{r}\right)_{0}}{\mu}=-\frac{p_{\infty}}{\mu}+\frac{1}{2} t^{4}+2 t-\frac{5}{2} .
$$


But $r_{i}^{\prime}=r_{0}^{2} / r_{i}^{2}=t^{2}$, and $V_{1}$ is given by (17), so we have

$$
\frac{r_{i}^{2}}{r_{0}^{2}} r_{1}^{\prime}+\frac{2 r_{i} r_{i}^{\prime}}{r_{0}^{2}} r_{1}=V_{1}=-\frac{p_{\infty}}{\mu}-\frac{3}{2}-\frac{1}{2} t^{4}+2 t
$$

This we can integrate using an integrating factor and the substitution used earlier, and we obtain

$$
r_{1}=\frac{c r_{0}^{3}}{r_{i}^{2}}+\frac{r_{0}^{4}}{2 r_{i}^{3}}+\frac{A}{r_{i}^{2}},
$$

where $A$ is an arbitrary constant and $c=-\frac{1}{2}-p_{\infty} /(3 \mu)$. It is the need to determine this constant $A$ that forces us to consider the next terms in the equation. In fact, since we have exactly matched $\sigma_{r} / \mu$ to its boundary values at leading order, when we integrate (28) from $a_{0}$ to infinity we must have

$$
\int_{a_{0}}^{\infty} L_{1}\left(r_{1}\right) d r_{0}=0
$$

and this is the linear equation that will determine $A$. In detail, when we substitute (17) into $L_{1}$ we obtain

$$
L_{1}\left(r_{1}\right)=-\frac{2 r_{1}}{r_{i}^{2}}+\frac{6 r_{0}^{6} r_{1}}{r_{i}^{8}}-\frac{4 r_{0}^{4} r_{1}^{\prime}}{r_{i}^{5}}
$$

where we have used (19b) again to eliminate $r_{i}^{\prime}$. Then when we substitute (39) into this we obtain

$$
L_{1}\left(r_{1}\right)=-\frac{2 c r_{0}^{3}}{r_{i}^{4}}-\frac{r_{0}^{4}}{r_{i}^{5}}-\frac{12 c r_{0}^{6}}{r_{i}^{7}}-\frac{8 r_{0}^{7}}{r_{i}^{8}}+\frac{14 c r_{0}^{9}}{r_{i}^{10}}+\frac{9 r_{0}^{10}}{r_{i}^{11}}-\frac{2 A}{r_{i}^{4}}+\frac{14 A r_{0}^{6}}{r_{i}^{10}}
$$

Then writing down (40), using the $t$-substitution and (32), we obtain

$$
\begin{aligned}
0 & =\int_{T}^{1}\left(\frac{2 c t^{3}+t^{4}+12 c t^{6}+8 t^{7}-14 c t^{9}-9 t^{10}}{t^{3}-1}+\frac{2 A}{B}-\frac{14 A t^{6}}{B}\right) d t \\
& =\int_{T}^{1}-2 c\left(t^{3}+7 t^{6}\right)-\left(t^{4}+9 t^{7}\right)+\frac{2 A}{B}\left(1-7 t^{6}\right) d t \\
& =\left[-2 c\left(\frac{t^{4}}{4}+t^{7}\right)-\left(\frac{9 t^{8}}{8}+\frac{t^{5}}{5}\right)+\frac{2 A}{B}\left(t-t^{7}\right)\right]_{T}^{1} \\
& =2 c\left(T^{7}+\frac{T^{4}}{4}-\frac{5}{4}\right)+\left(\frac{9 T^{8}}{8}+\frac{T^{5}}{5}-\frac{53}{40}\right)+\frac{2 A}{B}\left(T^{7}-T\right) .
\end{aligned}
$$

In the last term we can write $\left(T^{3}-1\right) / B=1 / a_{i}^{3}$, so the last term is $2 A\left(T^{4}+T\right) / a_{i}^{3}$, and finally we obtain

$$
A=-\frac{a_{i}^{3}}{T^{4}+T}\left\{c\left(T^{7}+\frac{T^{4}}{4}-\frac{5}{4}\right)+\left(\frac{9 T^{8}}{16}+\frac{T^{5}}{10}-\frac{53}{80}\right)\right\} .
$$

The resulting curves, plotted in Figure 3, are in good agreement with a fully nonlinear elastostatic calculation (carried out by Andrew Hazel). 


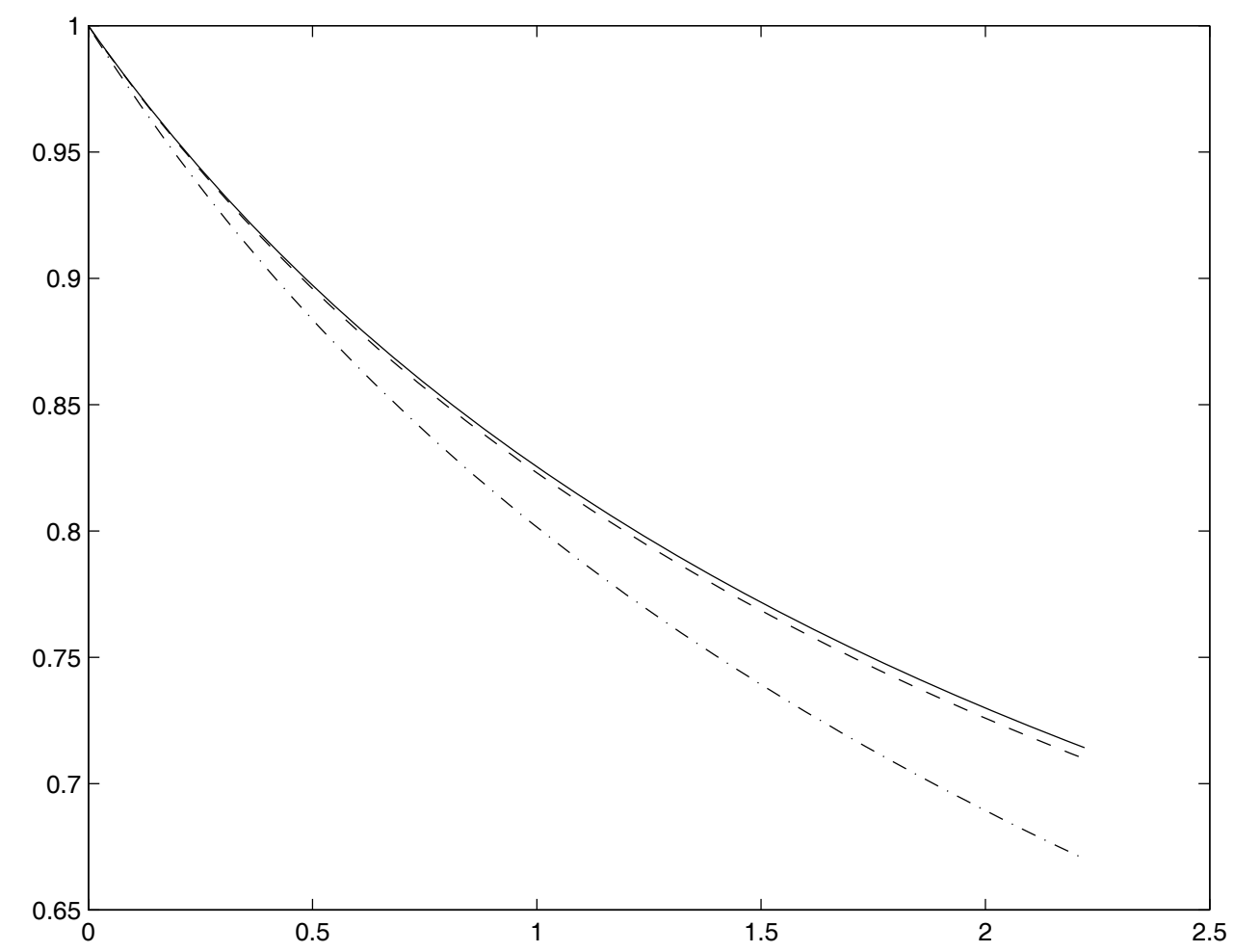

Figure 1: Compression ratio $a / a_{0}$ as a function of $p_{\infty} / \mu$ with $p_{\text {in }} / \mu=0$, for $\epsilon=0$ (solid), $\epsilon=10^{-2}$ (dashed) and $\epsilon=10^{-1}$ (dot-dashed).

\section{Scattering from the strained region}

The problem of acoustic scattering from a strained region may be solved via the technique of small displacements superposed on the finite deformation (otherwise known as "small on large" theory), using the stressed deformation as the reference configuration for the scattering problem, illustrated in figure 4 .

For our problem the specific configuration is shown in figure 4 , so that the cavity of initial radius $a_{0}$ in the configuration $B_{0}$ is finitely deformed via a pressure $p_{\infty}$ at infinity to a radius $a$ in the configuration $B$. For the purposes of the study group problem we are mainly interested in the monopole scattering characteristics since this is the dominant contribution to the scattering cross section of the cavity.

The section is divided into two parts. Firstly we analyse the problem formally by appealing to the "small on large" theory. However the complexity of this approach motivates a physical argument which leads to a more direct estimate of the monopole scattering cross section. 


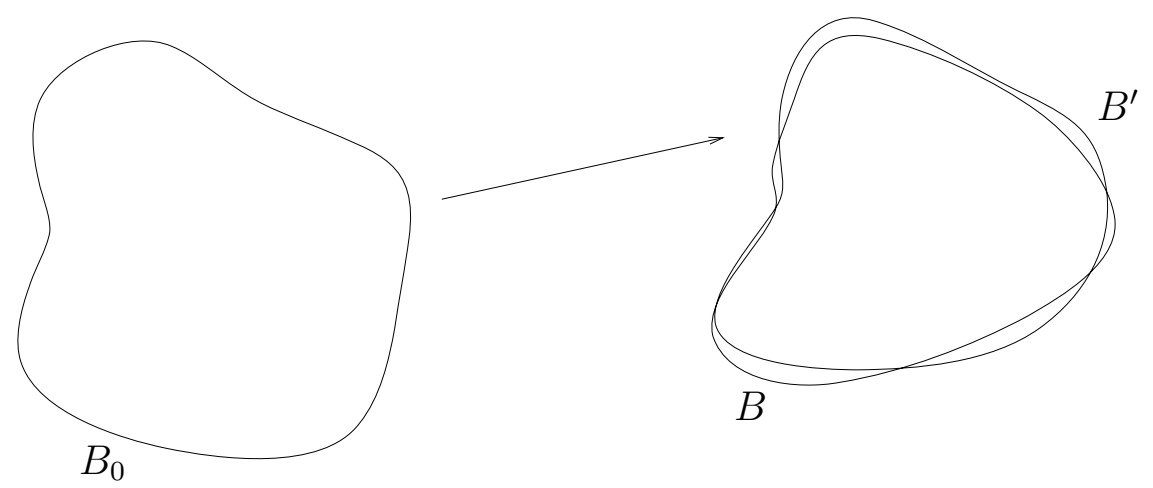

Figure 2: Figure to show the initial finite deformation from $B_{0}$ to $B$ and the small displacements superposed on $B$, deforming the material into the configuration $B^{\prime}$.

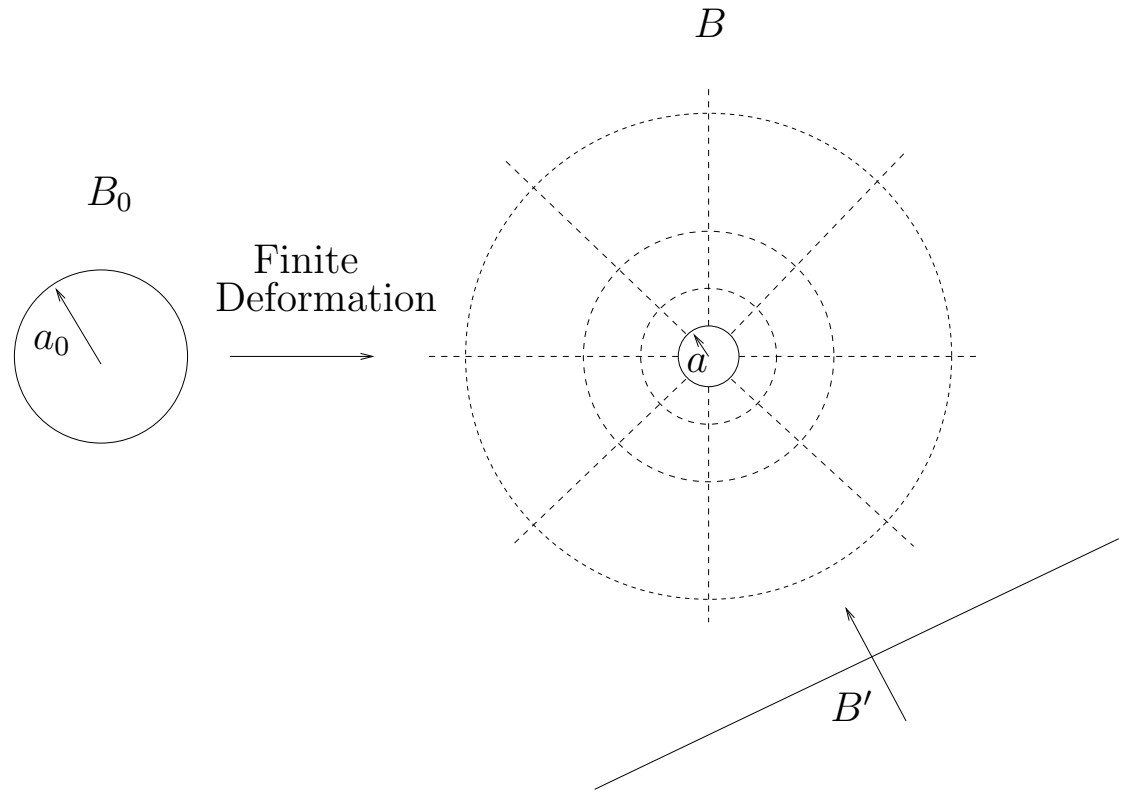

Figure 3: The configuration of the problem of scattering from a strained region around a spherical cavity. $B_{0}$ is the configuration of the undeformed material. $B$ is the configuration of the medium once the finite deformation has taken place (straining is represented by the dashed lines) and finally $B^{\prime}$ is the configuration of the strained medium with additional small deformations corresponding to the wave field.

\subsection{Formal Analysis of the Scattering Problem}

We will take $B$ as the reference state about which we linearize to find the equation of motion for the additional small displacements which correspond to the incident (and scattered) acoustic waves. 
We may use the "small on large" theory of Green and Zerna [4], (as was done in [1] for an initial incompressible finite deformation), in order to derive the full linear wave equations governing the scattered displacement field $\left(u_{r}, u_{\theta}\right)$ with the condition of axisymmetry:

$$
\begin{aligned}
A(r) \frac{\partial^{2} u_{r}}{\partial r^{2}}+B(r) \frac{1}{r^{2}} \frac{\partial^{2} u_{r}}{\partial \theta^{2}}+ & C(r) \frac{1}{r} \frac{\partial^{2} u_{\theta}}{\partial r \partial \theta}+\ldots \\
& +J(r) \frac{1}{r^{2}} u_{r}+K(r) \frac{\cot \theta}{r^{2}} u_{\theta}-\rho \frac{\partial^{2} u_{r}}{\partial t^{2}}=-F_{r}\left(r, \theta, \mathbf{u}_{\text {inc }}\right) \\
L(r) \frac{\partial^{2} u_{\theta}}{\partial r^{2}}+M(r) \frac{1}{r^{2}} \frac{\partial^{2} u_{\theta}}{\partial \theta^{2}}+ & N(r) \frac{1}{r} \frac{\partial^{2} u_{r}}{\partial r \partial \theta}+\ldots \\
& +U(r) \frac{1}{r^{2}} u_{r}+V(r) \frac{\csc ^{2} \theta}{r^{2}} u_{\theta}-\rho \frac{\partial^{2} u_{\theta}}{\partial t^{2}}=-F_{\theta}\left(r, \theta, \mathbf{u}_{\text {inc }}\right)
\end{aligned}
$$

where $A(r), B(r), \ldots, V(r)$ are coefficients which depend on the elastic constants of the material and the initial finite deformation (hence the $r$ dependence). The terms $F_{r}$ and $F_{\theta}$ are the forcing terms resulting from the differential operators on the left hand side of the equations operating on the incident displacement field $\mathbf{u}_{\text {inc }}$.

For the study group problem we were asked to try to understand the monopole scattering behaviour (due to an incident P-wave), since this was thought to be by far the strongest. The part of the scattered field associated with the monopole scattering will depend on $r$ only (and $t$ ) and since for axisymmetric problems $u_{\theta}$ cannot be a function only of $r$ its monopole contribution must be zero. Thus we can write the scattered displacement in the form

$$
\begin{aligned}
& u_{r}(r, \theta, t)=U(r, t)+\text { higher order scattering, } \\
& u_{\theta}(r, \theta, t)=0+\text { higher order scattering, }
\end{aligned}
$$

where $U(r, t)$ is associated with the monopole scattering. Thus we are looking for that part of the solution of (48) and (49) which depends on $r$ (and $t$ ) only.

If we consider an incident plane wave of the form

$$
\mathbf{u}_{\mathrm{inc}}=u_{1} \mathbf{e}_{z} \exp i(\omega t-k z),
$$

then the stresses in this are

$$
\left(\sigma_{m n}^{\mathrm{inc}}\right)=\left(\begin{array}{ccc}
-\lambda^{\prime} i k u_{1} & 0 & 0 \\
0 & -\lambda^{\prime} i k u_{1} & 0 \\
0 & 0 & -\left(\lambda^{\prime}+2 \mu\right) i k u_{1}
\end{array}\right) e^{i(\omega t-k z)},
$$

where $\lambda^{\prime}$ is the effective Lamé modulus of the material modified by the static pressure $p_{\infty}$. (This is in fact $\lambda^{\prime}=\lambda-p_{\infty}+O(\epsilon \mu)$ when $p_{\infty}=O(\mu)$ as we consider.) Since $\lambda^{\prime} \gg \mu$, this stress tensor is, at leading order, just isotropic,

$$
\sigma_{m n}^{\text {inc }}=\left(-\lambda i k u_{1}+O(\mu)\right) \delta_{m n} e^{i(\omega t-k z)} .
$$

As we are interested in the low frequency regime $(a \ll \lambda)$ we have $k a \ll 1$, and so $\exp (-i k z)$ can be approximated by 1 in the scattering region. (We note that for $p_{\infty}$ of 
order $\mu$, the size of the region where significant strain occurs around the microsphere is of order a.) We therefore approximate the incident forcing responsible for monopole scattering as

$$
\sigma_{r r}^{\mathrm{inc}}=-\lambda i k u_{1} e^{i \omega t}
$$

If we then write the radial displacement in the monopole scattered field as

$$
U(r, t)=u_{1} U(r) e^{i \omega t}
$$

we find that

$$
A(r) \frac{\partial^{2} U}{\partial r^{2}}+D(r) \frac{1}{r} \frac{\partial U}{\partial r}+\left(J(r) \frac{1}{r^{2}}+\rho \omega^{2}\right) U=-\left(J(r) \frac{1}{r^{2}}+\rho \omega^{2}\right) .
$$

Here $U(r)$ is a scaled displacement of course, entirely distinct from the elastic coefficient $U(r)$ in (49). In order to determine the coefficients $A(r), D(r)$ and $J(r)$ we should use the solution of the slightly compressible finite deformation problem found above. It is clear that the coefficients $A(r), D(r)$ and $J(r)$ should then tend to those of the linear Navier equations with an additional pressure of that imposed at infinity (and hence a pre-stressed substrate). Thus as $r \rightarrow \infty$ we should find that

$$
\begin{array}{r}
A(r) \rightarrow 2 \mu+\lambda-p_{\infty}, \\
D(r) \rightarrow 2\left(2 \mu+\lambda-p_{\infty}\right), \\
J(r) \rightarrow-2\left(2 \mu+\lambda-p_{\infty}\right) .
\end{array}
$$

These coefficients can be found as a function of the principal stretches given in equations (13) and (14) above and using the approach in [1] and [4]. Unfortunately due to time constraints these coefficients were not found during the study group but certain contributors hope to address this problem shortly.

Note also that the shear modulus appearing in these coefficients is the dynamic shear modulus and referring to the discussion of constitutive behaviour above, it is reasonable to directly replace the static shear modulus $\mu$ by the dynamic shear modulus $\mu(\omega)$ for a single cavity in an infinite medium.

Once the coefficients $A(r), D(r)$ and $J(r)$ have been found, (57) can be solved either numerically or via asymptotic matching in order to find the scattered field $U(r, t)$ and hence the monopole scattering coefficient is obtained:

$$
\gamma_{\text {monopole }}=\frac{\text { energy scattered (via monopole) per unit time }}{\text { energy per unit area carried by incident wave per unit time }}
$$

where for the monopole contribution the rate at which energy is being scattered is

$$
\iint \Sigma_{r r} \frac{\partial U}{\partial t} d S
$$

where $S$ is some spherical surface concentric with the cavity, having a radius greater than that of the cavity, and $\Sigma_{r r}$ is the additional stress due to the monopole scattered field. 


\subsection{Physical Arguments}

Instead of the formal approach used in the previous section, we may repeat the physical argument given in [1] but now use the slightly compressible initial deformation and the dynamic shear modulus $\mu(\omega)$.

With reference to figure 4.2, we suppose that all the stress induced anisotropy (the strained region) is located in a region local to the cavity, characterised by a lengthscale $L$ which although much greater than the deformed radius $a$ of the cavity is still much less than the wavelength of the incident wave $\lambda$. We suppose that the exterior of this region remains isotropic.

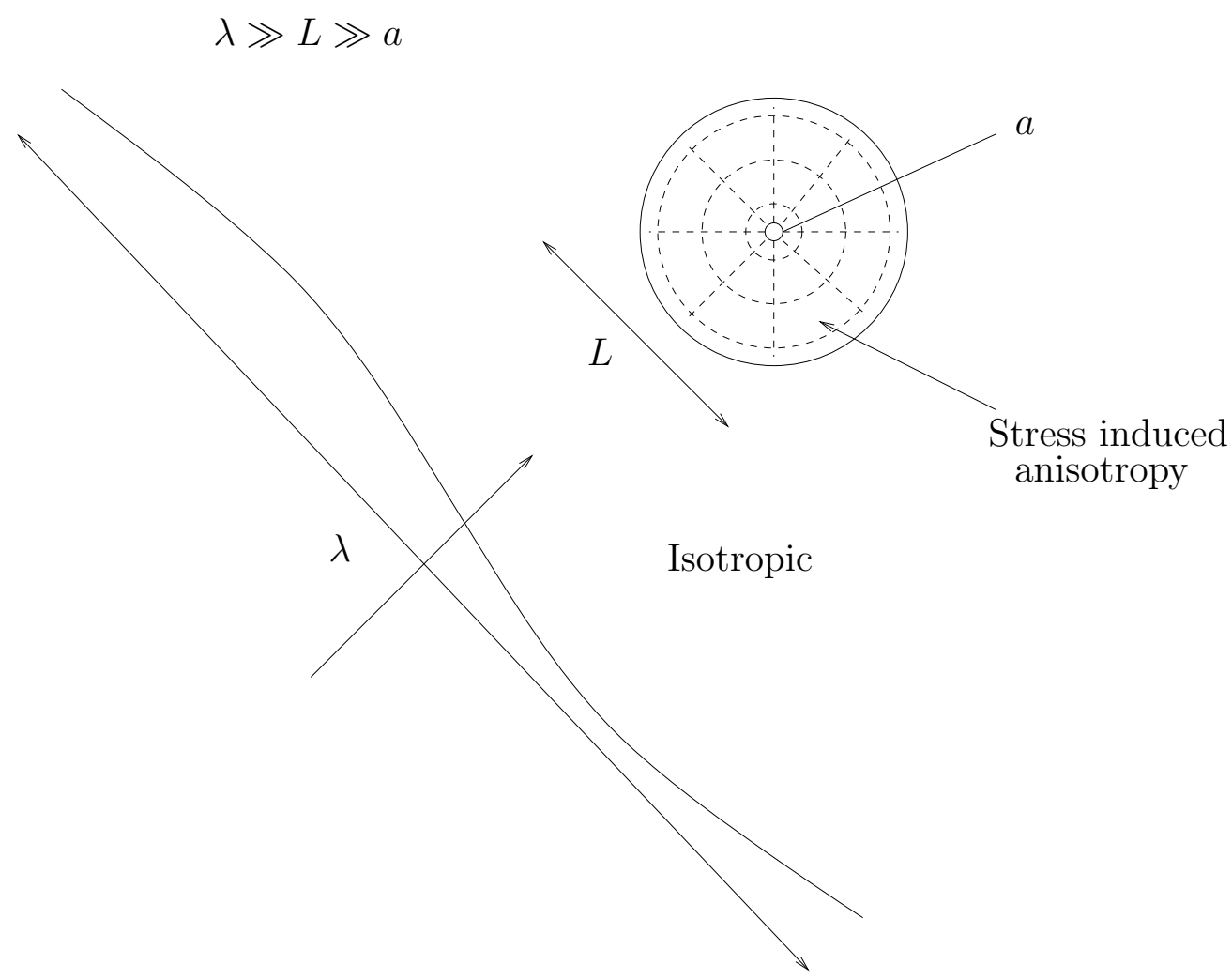

Figure 4: Illustration of the geometry of the problem and the lengthscales involved in the physical argument.

It is clear that monopole behaviour relates to volume changes. On considering an incident field of the form (52-53) the cavity will see (at leading order) the pressure field

$$
p=p_{s}+p_{1} \cos (\omega t-k z)
$$

where $p_{s}$ is the steady static pressure and $p_{1}$ is the acoustic amplitude. Referring once more to figure 4.2, for low frequencies the inner region will be subjected to a slowly varying static pressure and since $\lambda \gg L$ it will respond quasi-statically, since to leading order it will see only the pressure field

$$
p=p_{s}+p_{1} \cos (\omega t) .
$$


Thus the radius of the compressed cavity varies according to:

$$
a=a_{s}+a_{1} \cos (\omega t)
$$

where $a_{s}$ is the compressed radius of the micro-sphere under the static pressure $p_{s}$ and

$$
a_{1}=\left.\left(\frac{d a}{d p}\right)\right|_{p_{s}} \frac{1}{3} p_{1}
$$

which is small and therefore the volume fluctuation is approximately $V(t) \approx$ $4 \pi a_{s}^{2} a_{1} \cos (\omega t)$.

Since the outer region is approximately isotropic it responds like an acoustic medium to the volume fluctuation and thus the radiation of energy is (Lighthill [5])

$$
E_{R}=\frac{\rho(\ddot{V})^{2}}{4 \pi c_{p}}
$$

Now define

$$
E_{S}=\int_{0}^{2 \pi / \omega} E_{R} d t=\frac{2 \pi \rho a_{s}^{4} \omega^{4} p_{1}^{2}}{c_{p}}\left(\left.\left(\frac{d a}{d p}\right)\right|_{p_{s}}\right)^{2}
$$

which is the time averaged radiated energy.

The incident energy flux density is

$$
E_{I}=\frac{p_{1}^{2}}{2 \rho c_{p}}
$$

and thus

$$
\gamma_{\text {monopole }} \sim \frac{4 \pi^{2}}{\rho} a_{s}^{4} \omega^{4}\left(\left.\left(\frac{d a}{d p}\right)\right|_{p_{s}}\right)^{2}
$$

In [1], both $a_{s}$ and $d a /\left.d p\right|_{p_{s}}$ were calculated via the incompressible solution of the static problem and with the static shear modulus $\mu$. They may now be calculated via the small compressibility static solution obtained above. Furthermore we may directly replace $\mu$ by $\mu(\omega)$, the dynamic shear modulus for scattering by a single spherical cavity.

\section{Conclusions}

In this study group report we have considered the problem of acoustic scattering from a strained region where the shear modulus is considered frequency dependent. We argued that since the material exterior to the strained region is homogeneous we may simply replace the static shear modulus with the dynamic one given in (5). Different models 
are, however, needed for filled rubbers and this could be significant in "real" materials where we may not be able to neglect interaction effects between inclusions (cavities).

The initial static problem (originally considered in [1]) was extended in order to include small compressibility, a notion essential so that compressive waves can travel through the material. The "next order" terms were found for the deformed radius and we compared values of the compression ratio $a / a_{0}$ for a range of $p_{\infty} / \mu$ obtained via $\epsilon=0$ (the incompressible case) with various compressible solutions with $\epsilon \ll 1$.

We considered two approaches to the scattering problem. The first is the formal approach using the "small on large" theory. Restricting attention to monopole scattering enabled us to consider a simplified (although still complicated!) equation (57), the coefficients of which depend on the (corrected) static problem allowing for small compressibility. The complexity of this problem motivated a physical argument in order to deduce an expression for the monopole scattering coefficient which was given in (70). This expression is a function of $a_{s}$ and $d a /\left.d p\right|_{p_{s}}$ which should now be calculated via the solution to the slightly compressible static deformation solved above (with dynamic shear modulus) rather than using the incompressible solution obtained in [1].

\section{References}

[1] Elastic scattering from a strained region. MSc dissertation of W.J.Parnell. http://eprints.maths.ox.ac.uk/archive/00000014/.

[2] On a fully three-dimensional finite-strain viscoelastic damage model: formulation and computational aspects. J.C.Simo. Computer methods in applied mechanics and engineering 60, 153-173, 1987.

[3] A constitutive model and FEA of rubber under small oscillatory load superimposed on large static deformation. B.-K. Kim, S.-K. Youn, and W.-S. Lee. Archive of applied mechanics 73, 781-798, 2004.

[4] Theoretical elasticity. A.E.Green and W.Zerna. 2nd edition, OUP, 1968.

[5] Waves in fluids. James Lighthill. CUP, 1978. 